\title{
HIGH-RESOLUTION AND FAST 3D ULTRASONIC IMAGING TECHNIQUE
}

\author{
Zalman M. Benenson, Alexey B. Elizarov, Tatiana V. Yakovleva \\ Scientific Council on Cybernetics, Russian Academy of Sciences, \\ Vavilova st. 40, GSP-1, Moscow, 119991, Russia \\ William D. O’Brien, Jr. \\ Bioacoustics Research Laboratory, Department of Electrical and Computer Engineering, University of Illinois, \\ 405 N. Mathews, Urbana, IL 61801 U.S.A
}

Abstract - The paper considers a 3D ultrasound imaging technique based upon usage of a probe that consists of 2 one-dimensional transmitting phased arrays and 3 receiving phased arrays. By controlling the transmit process on the elements of the transmitting array and simultaneous receiving the signals by all the elements of the receiving arrays, this method yields algorithmically synthesized signals of a 2D phased array, the so called virtual 2D phased array. This allows for achieving short volume scan time and high lateral and axial resolution. Lateral resolution is 2 times better than the Rayleigh limit of the original $1 \mathrm{D}$ phased arrays. The paper contains a mathematical simulation and experiment results.

\section{INTRODUCTION}

Three-dimensional acoustical imaging is of significant practical importance both for medical ultrasound diagnostics and for nondestructive evaluation. At the present time implementation of 3D imaging is based upon two principle techniques. The first technique consists of mechanically scanning the transducer with an annular array along azimuth and elevation angles [1]. The significant disadvantage of this method is the long time required for scanning the total volume (order of 2-3 sec.). The second technique is realized by means of linear or convex arrays being mechanically moved perpendicular to the electronic scan plane or by rotation around the longitudinal axis of the array [2]. The total scan time in this technique is also long and the lateral resolution in the motion plane is poor.

Fast electronically scanning at 2 angles with dynamical focusing can be implemented with a 2D phased array. However, fabrication of such an array at present time for practical application is rather doubtful and the complexity of the hardware is high, especially for high frequencies of 7-20 MHz [3].
The current paper considers a technique of electronically scanning the 3D volume that utilizes 2 transmitting and 3 receiving $1 \mathrm{D}$ arrays. The emission of the signals is executed sequentially while the reception of the reflected signals is performed simultaneously.

\section{2D VIRTUAL PHASED ARRAY AND THE SIGNAL PROCESSING ALGORITHM}

Fig. 1 shows the layout of 5 arrays. By processing their signals one obtains signals of a 2D virtual phased array. The structure consists of 3 receiving arrays $P_{H}$ and 2 transmitting ones $P_{v}$. Each of them has a concave cylindrical lens with a near focus $F$ installed, to broaden the radiation pattern along the $Y$-axis for $P_{H}$ and along the $X$-axis for $P_{v}$. Elements of the arrays $P_{v}$ sequentially emit pulses with repetition period $T_{0}=\frac{2 z_{\max }}{c} \quad\left(z_{\max }\right.$ is the maximum depth of the medium, $c$ is the sound speed). The reflected signals are received by all the

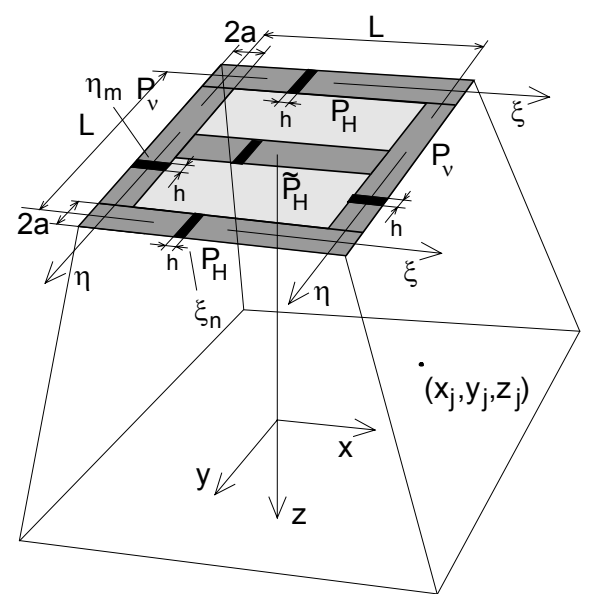

Fig. 1. The layout of 5 onedimensional phased arrays. 
elements of the arrays $P_{H}$ simultaneously.

First, let us derive a theory for the probe consisting of 4 arrays (central receiving array $\tilde{P}_{H}$ is excluded, see Fig. 1).

The radiation pattern of the $m$-th element of the transmitting array at $y=\eta_{m}$ with Gaussian apodization function at the aperture of size $2 a$, taking into account the $P_{v}$ arrays shift $\xi_{\mp N}$, at the frequency $\omega$ is as follows (in the Fresnel approximation):

$$
\begin{aligned}
D_{t r}\left(x_{j}-\xi_{\mp N}, y_{j}-\eta_{m}, z_{j}, \omega\right)=A_{1} \times \\
\quad \times \exp \left(-\frac{\left(x_{j}-\xi_{\mp N}\right)^{2}}{\alpha-i \zeta}\right) \exp \left(-\frac{\left(y_{j}-\eta_{m}\right)^{2}}{\alpha_{1}-i \zeta_{1}}\right),
\end{aligned}
$$

where

$$
\begin{aligned}
& \alpha=\frac{1}{4 / a^{2}+(\omega a / c F)^{2}} \approx \frac{c^{2} F^{2}}{\omega^{2} a^{2}}, \\
& \zeta=\frac{c}{2 \omega}\left(-\frac{F}{1+4\left(c F / \omega a^{2}\right)^{2}}-z\right) \approx-\frac{c}{2 \omega}(z+F), \\
& \alpha_{1}=\frac{h^{2}}{16}, \zeta_{1}=-\frac{c}{2 \omega} z, h \text { - array step. }
\end{aligned}
$$

The approximation is valid for $a>2 \lambda$ and $a / F \sim 1$.

The radiation patterns of the receiving arrays $D_{r e}\left(x_{j}-\xi_{n}, y_{j}-\eta_{\mp N}, z_{j}, \omega\right)$ have analogous form (the $x, \xi$ axes are exchanged for $y, \eta$ ).

The received signal set for a set of point reflectors with coordinates $x_{j}, y_{j}, z_{j}$ at the receiving array elements $\xi_{n}$ for emission from the $\eta_{m}$ elements of the transmitting arrays in frequency domain $\omega$ has the form:

$$
\begin{aligned}
S_{0}^{(\mp N, \mp N)} & \left(\omega, \xi_{n}, \eta_{m}\right)=\sum_{j} \gamma_{j} \times \\
& \times D_{t r}\left(x_{j}-\xi_{\mp N}, y_{j}-\eta_{m}, z_{j}, \omega\right) \times \\
& \times D_{r e}\left(x_{j}-\xi_{n}, y_{j}-\eta_{\mp N}, z_{j}, \omega\right) \times \\
& \times E(\omega) \exp \left(i \frac{\omega}{c} 2 z_{j}\right) \\
& (n, m=-N, \ldots, 0, \ldots, N),
\end{aligned}
$$

where $(\mp N, \mp N)$ defines a combination of the signals of the corresponding pair of the $P_{H}$ and $P_{\mathrm{v}}$ arrays, $\eta_{m}$ and $\xi_{n}$ are the coordinates of the array elements, $E(\omega)$ is the Fourier transform of the emitted ultrasound pulse, $\gamma_{j}$ is the reflection coefficient of the $j$-th point reflector.

Then let us algorithmically form subapertures of size $2 a$ with negative focus $F$ on the arrays by implementing a 2D convolution of the received signals in the frequency domain with a complexvalue weight function $K\left(\xi_{n}, \eta_{m}, \omega\right)=\exp \left(-\left(\frac{1}{a^{2}}-i \frac{\omega}{2 c F}\right)\left(\xi_{n}^{2}+\eta_{m}^{2}\right)\right) . \quad$ The expression for the resulting signal is:

$$
\begin{aligned}
S^{(\mp N, \mp N)} & \left(\omega, \xi_{n}, \eta_{m}\right)=\sum_{j} \gamma_{j} A_{2} \\
& \times \exp \left(-\frac{\left(x_{j}-\xi_{n}\right)^{2}+\left(x_{j}-\xi_{\mp N}\right)^{2}}{\alpha-i \zeta}\right) \times \\
& \times \exp \left(-\frac{\left(y_{j}-\eta_{m}\right)^{2}+\left(y_{j}-\eta_{\mp N}\right)^{2}}{\alpha-i \zeta}\right) \times \\
& \times E(\omega) \exp \left(i \frac{\omega}{c} 2 z_{j}\right) \\
& (n, m=-N, \ldots, 0, \ldots, N),
\end{aligned}
$$

It follows from (3) that we have the set of signals $S^{(\mp N, \mp N)}(\bullet)$ that consists of 4 subsets, the signals depending on coordinates of the array elements $\xi_{n}$, $\eta_{m}$. Using some simple transforms, these sets of signals can be combined to a single set of signals $\tilde{S}\left(\omega, \hat{\xi}_{n}, \hat{\eta}_{m}\right)$ of some $2 \mathrm{D}$ virtual array with the following coordinates of the elements:

$$
\begin{aligned}
& \hat{\xi}_{\hat{n}}=\frac{\xi_{n}}{2}-\frac{L}{4}, \hat{n}=n-N\left(\hat{\xi}_{\hat{n}}<0\right) ; \\
& \hat{\xi}_{\hat{n}}=\frac{\xi_{n}}{2}+\frac{L}{4}, \hat{n}=n+N\left(\hat{\xi}_{\hat{n}} \geq 0\right) ; \\
& \hat{\eta}_{\hat{m}}=\frac{\eta_{m}}{2}-\frac{L}{4}, \hat{m}=m-N\left(\hat{\eta}_{\hat{m}}<0\right) ; \\
& \hat{\eta}_{\hat{m}}=\frac{\eta_{m}}{2}+\frac{L}{4}, \hat{m}=m+N\left(\hat{\eta}_{\hat{m}} \geq 0\right) .
\end{aligned}
$$


One can easily see that the number of the $2 \mathrm{D}$ virtual array elements is equal to $16 N^{2}$ (by each coordinate it is twice the number of elements of the original arrays). Because the emitted pulse is short and its frequency band is approximately equal to the carrier frequency, the signals (3) can be obtained as a result of the Fourier transform by time $t$. However, this brings uncertainty in the $z$ coordinate. For this reason the virtual array signals $\tilde{S}_{l}(\bullet)$ are calculated for a set of hypotheses of the $z$ coordinate value. The whole depth range is divided into $M$ adjacent intervals $\left[z_{l}, z_{l+1}\right]$ to provide the required focusing accuracy.

The transform to obtain the signals $\tilde{S}_{l}(\bullet)$ at the corresponding depth intervals looks as follows:

$$
\begin{aligned}
& \tilde{S}_{l}\left(\omega, \hat{\xi}_{\hat{n}}, \hat{\eta}_{\hat{m}}\right)=S^{(\mu N, v N)}\left(\omega, \xi_{n}, \eta_{m}\right) \times \\
& \times \exp \left(\frac{\left(\delta_{n}^{(i)}\right)^{2}}{8\left(\alpha-i \zeta_{l}\right)}\right) \exp \left(\frac{\left(\delta_{m}^{(j)}\right)^{2}}{8\left(\alpha-i \zeta_{l}\right)}\right),
\end{aligned}
$$

where $\mu="-", i=1$ for $\hat{\xi}_{\hat{n}}<0 ; \mu="+", i=2$ for $\hat{\xi}_{\hat{n}} \geq 0 ; v="-", j=1$ for $\hat{\eta}_{\hat{m}}<0 ; v="+", j=2$ for $\quad \hat{\eta}_{\hat{m}} \geq 0 ; \quad \delta_{n}^{(1,2)}=\mp(L-2 a) / 2-\xi_{n}$, $\delta_{m}^{(1,2)}=\mp(L-2 a) / 2-\eta_{m}$. The exponential functions of $\delta_{n}^{(1,2)}$ and $\delta_{m}^{(1,2)}$ account for differences between coordinates of the transmitting and receiving elements.

Finally, for the set of point reflectors with coordinates $x_{j}, y_{j}, z_{j}$ and reflection coefficients $\gamma_{j}$ we get the set of the virtual array signals of the form:

$$
\begin{aligned}
& \tilde{S}\left(\omega, \hat{\xi}_{n}, \hat{\eta}_{m}\right)=\sum_{j} \gamma_{j} \times \\
& \times \exp \left(-\frac{\left(x_{j}-\hat{\xi}_{n}\right)^{2}+\left(y_{j}-\hat{\eta}_{m}\right)^{2}}{2(\alpha-i \zeta)}\right) .
\end{aligned}
$$

After focusing by the algorithm based on the 3D Fourier transform [4], the resolution at far distances will be about $\lambda z / 2 L$, i.e., 2 times higher than that in a conventional technique of dynamic focusing on receiving by a phased array.

The drawback of the 4-array probe is that the virtual array formed according to (4) has a hole at the center by the $\hat{\eta}$ coordinate of size $2 a$. This results in a high side lobe level. To fill the hole, an additional receiving array $\tilde{P}_{H}$ is used. This allows for limiting the hole to a square of $2 a \times 2 a$ size at the center of the virtual array, and gives acceptable side lobe level. The corresponding received signal set is designated as $S_{0}^{(\mp N, 0 N)}\left(\omega, \xi_{n}, \eta_{m}\right), \eta_{0 N}=0$. The transform (5) then is extended to allow index $\mu=" 0 ", i=0$, $\delta_{m}^{(0)}=-\eta_{m}$ for the virtual array data that cannot be obtained from other 4 sets of source signals.

The signal processing algorithm consists of the following stages: fast Fourier transform by time of the received signals; forming subapertures from the original array elements (3); dividing the depth range into intervals $\left[z_{l}, z_{l+1}\right]$; forming the virtual array signals $\tilde{S}_{l}$ (5); forming the signals $\tilde{S}$; implementing the focusing algorithm based upon 3D Fourier transforms [4]; visualization of the 3D image.

\section{RESULTS OF EXPERIMENTS}

The proposed scheme and the algorithm were mathematically simulated and tested on digitized data obtained from an ultrasound phantom of physical medium.

Fig. 2 presents results of the mathematical
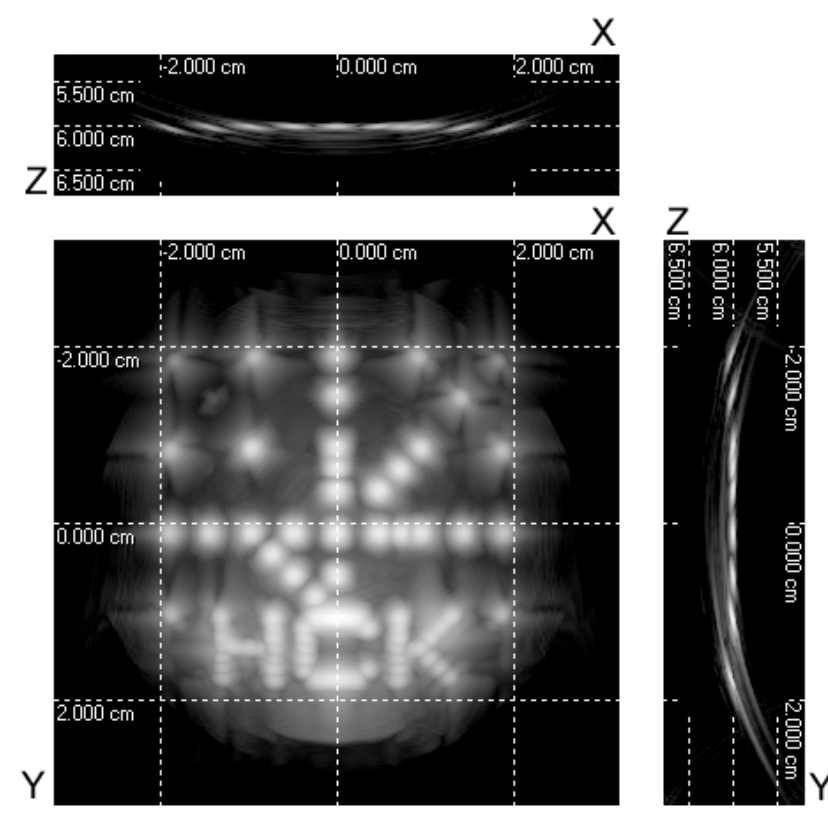

Fig. 2. Mathematical simulation results for a set of point reflectors in a 3D volume.

Dynamic range $60 \mathrm{~dB}$. 


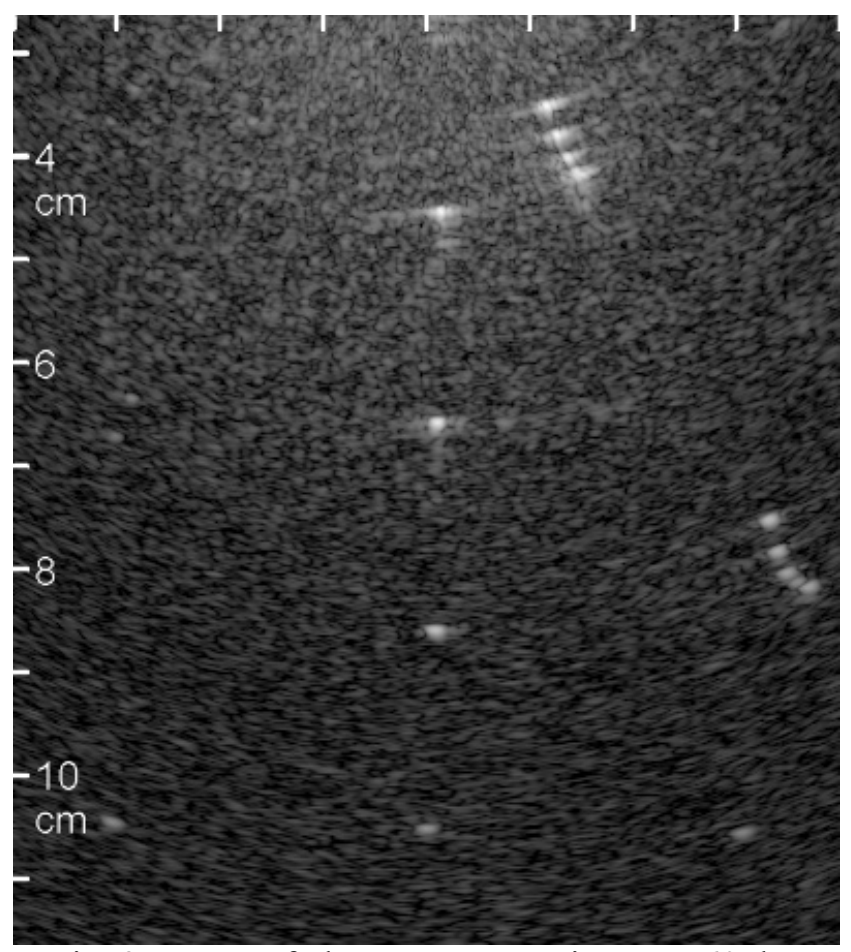

Fig. 3. Image of phantom. Dynamic range $60 \mathrm{~dB}$.

simulation. The figure shows cross-sections of a 3D image obtained as a result of reflection of ultrasound waves at the center carrier frequency of $f=3.0 \mathrm{MHz}$ and frequency band of $3 \mathrm{MHz}$ from a set of given sample point reflectors and nylon wires arranged to form the "HCK" letters placed at fixed distances. The image shows an area at $6 \mathrm{~cm}$ depth. The probe frame size is $1.6 \mathrm{x} 1.6 \mathrm{~cm}$, the arrays' widths are $0.15 \mathrm{~cm}$. In the $\mathrm{XZ}$ and $\mathrm{YZ}$ sections one has no possibility to reconstruct the factual image. Meanwhile, widely used 2D ultrasound imaging devices produce these cross-sections. In the XY plane the image structure is clear. The simulated results yielded a lateral resolution from $0.11 \mathrm{~cm}$ at $3.0-\mathrm{cm}$ depth to $0.56 \mathrm{~cm}$ at $15-\mathrm{cm}$ depth, an axial resolution of $0.05 \mathrm{~cm}$ (at -10 $\mathrm{dB}$ level), a side lobe level of $-25 \mathrm{~dB}$; the whole volume sector of $60 \times 60$ degrees started from $z=3.0 \mathrm{~cm}$; the frame rate was $39 \mathrm{fps}$.

In the physical experiment to test the algorithm of the virtual array construction for 2D case we used the data obtained at Biomedical Ultrasonics Laboratory, University of Michigan (http://bul.eecs.umich.edu). Linear phased array radiated ultrasound to a phantom in a biological medium; the array length was $2.8 \mathrm{~cm}$, center carrier frequency was $3.5 \mathrm{MHz}$. Fig. 3 represents an image of the phantom cross-section. The width of the nylon wires of the phantom at the $-10 \mathrm{~dB}$ level ranged from $0.1 \mathrm{~cm}$ at $z=4.5 \mathrm{~cm}$ to
$0.17 \mathrm{~cm}$ at $z=10.0 \mathrm{~cm}$. This result is in good agreement with theoretical estimates $\frac{\lambda z}{2 L}$ (half a Rayleigh limit).

\section{CONCLUSION}

The proposed design of the probe that consists of $51 \mathrm{D}$ phased arrays and the theory of 2D virtual array signal synthesis allow for 3D imaging with high speed of electronic scanning and lateral resolution improvement 2 times compared to Rayleigh limit.

\section{ACKNOWLEDGEMENTS}

The work was supported by grant 02-01-01087 from the Russian Foundation of Basic Research (RFBR) and RP2-2104 from the U.S. Civilian Research and Development Foundation (CRDF).

\section{REFERENCES}

[1] D. Kirbach, T. A. Whitlingham. 3D Ultrasound - the Kretztechnic Voluson ${ }^{\circledR}$ Approach. Eur. J. Ultrasound, vol. 1, pp. 85-89, 1994.

[2] Thomas R. Nelson and Dolores H. Pretorius. Three-Dimensional Ultrasound Imaging. Ultrasound Med. Biol., vol.24, N9, pp. 12431270, 1998.

[3] S. W. Smith, W. Lee, E. D. Light, J. T. Yen, P. Wolf, S. Idriss. Two Dimensional Arrays for 3D Ultrasound Imaging. Proc. IEEE Ultrason. Symp. 2002, pp. 1509-1517, 2002.

[4] Z. M. Benenson, A. B. Elizarov, T. V. Yakovleva, and W. D. O’Brien, Jr. Approach to 3D Ultrasound High Resolution Imaging for Mechanically Moving Large-Aperture Transducer Based upon Fourier Transform. IEEE Trans. Ultrason., Ferroelect., Freq. Contr., vol. 49, no. 12, pp. 1665-1685, 2002. 